\title{
Fluctuation of Conductance Peak Spacings in Large Semiconductor Quantum Dots
}

\author{
Yshai Avishai ${ }^{1,2}$, Daniel Berend ${ }^{2}$, and Richard Berkovits ${ }^{3}$ \\ ${ }^{1}$ Department of Physics, Ben-Gurion University of the Negev, Beer-Sheva 84105, Israel \\ ${ }^{2}$ Department of Mathematics and Computer Sciences, Ben-Gurion University of the Negev, \\ Beer-Sheva 84105, Israel \\ ${ }^{3}$ Minerva Center, Department of Physics, Bar Ilan University, Ramat Gan 52900, Israel
}

Fluctuation of Coulomb blockade peak spacings in large two-dimensional semiconductor quantum dots are studied within a model based on the electrostatics of several electron islands among which there are random inductive and capacitive couplings. Each island can accommodate electrons on quantum orbitals whose energies depend also on an external magnetic field. In contrast with a single island quantum dot, where the spacing distribution is close to Gaussian, here the distribution has a peak at small spacing value. The fluctuations are mainly due to charging effects. The model can explain the occasional occurrence of couples or even triples of closely spaced Coulomb blockade peaks, as well as the qualitative behavior of peak positions with the applied magnetic field. 


\section{INTRODUCTION}

Recently, it became apparent that the physics exposed in the addition spectra of quantum dots is rather rich, and hence its investigation is at the focus of both experimental and theoretical studies. The present work concentrates on the distribution of spacings between Coulomb blockade peaks in large semiconductor quantum dots. Coulomb blockade is evidently one of the hallmarks of mesoscopic physics. The experimental achievement of tracing an addition of a single electron to a quantum dot and the appearance of isolated conductance peaks led to the concept of single electron transistors. After the origin of Coulomb blockade peaks has been elucidated, investigation is directed toward more subtle questions like their heights, widths and spacings. The underlying physics is related to the ground state energy, chemical potential and inverse compressibility of a few electron islands coupled capacitively to its environment, as well as fluctuations of these quantities with the number $N$ of electrons on the dot.

As far as the distribution of spacings between adjacent Coulomb blockade peaks is concerned, the question can be stated as follows: According to the simplest picture in which the quantum dot is regarded as a single electron island whose coupling with the leads is through its capacitance $C$, the total potential energy of a quantum dot is $Q^{2} / 2 C-V_{g} Q$, where $V_{g}$ is the corresponding gate voltage. The conductance peaks occur at those values of $V_{g}$ for which $C V_{g}=e(N+1 / 2)$, where $e$ is the electron charge (henceforth $e=-1$ ). For this value of $V_{g}$ the addition of an electron to the dot (which contains $N$ electrons) does not cost any charging energy. The position of the $N^{\text {th }}$ Coulomb blockade peak is then a linear function of $N$, and therefore the spacing should be a constant $1 / C$, independent of $N$. Recent experiments [1,2] indicate however that spacing between Coulomb blockade peaks in small quantum dots is in general not constant but, rather, a fluctuating quantity close to Gaussian. The average of

its distribution approximately coincides with the constant value mentioned above, but the elucidation of its standard deviation is still under investigation [3, 4].

The situation is even more intriguing if the quantum dot is very large. As indicated in a series 
of recent experiments, the spacing occasionally vanishes, namely, two peaks (and sometimes even three peaks) coincide. Moreover, the evolution of peak positions and spacings with an applied magnetic field indicates the existence of strong correlations between them [5-7]. These observations motivated numerous theoretical models based on the concept of pair tunneling [8] or that of two-electron bound-states in depleted electron islands [9].

In the present work we examine the scenario according to which a large quantum dot, like the one used in the last experiment [7], is, in fact, composed of several electron islands which are coupled capacitively among themselves as well as to the leads. Electrons are added in such a way that the total potential energy of the dot is minimum. This simple generalization of the single island picture leads to a remarkable change in the spacing distribution from a Gaussian [1,3,4] centered around a finite average to one which is large at small spacings. When the coupling between islands is zero, the distribution has indeed a maximum at zero spacing. If the coupling is non-zero but small, the maximum is slightly shifted away from zero, yet leaving an appreciable tail down to zero. These results are short of explaining the perfect overlap of peaks, since it requires a delta function component at zero spacing. Yet, it leads to the occurrence of couples and sometimes triples of closely spaced peaks, similar to the experimental observation. Moreover, the evolution of the peak positions with the magnetic field is qualitatively similar to the experimental one. On the other hand, the present model does not predict a definite periodicity in the bunching of Coulomb blockade peaks with electron number $N$. In the next section the model is explained, and the results of the calculations are presented in section 3 .

\section{FORMALISM}

We consider a large isolated two-dimensional quantum dot in a perpendicular magnetic field $B$ subject to a gate voltage $V_{g}$. Unlike the traditional Coulomb blockade picture, it might contain several electron islands which can be regarded as metallic objects with inductive couplings among themselves. These are determined by a positive definite symmetric 
matrix $C$ whose diagonal elements $C_{i i} \equiv C_{i}$ - the corresponding capacities - are positive, whereas the nondiagonal elements $C_{i j}=C_{j i},(i \neq j)$ - the corresponding coefficients of induction - are negative. The electrostatic energy of such a system may be written as

$$
E_{c}=\frac{1}{2} \sum_{i, j=1}^{K} p_{i j} N_{i} N_{j}-V_{g} N
$$

where $N_{i}$ is the number of electrons on island $i$ (the number of islands $K>1$ might be around 10), $N=\sum_{i=1}^{K} N_{i}$ is the total numbers of electrons, and $p=\left(p_{i j}\right)$ is the (symmetric positive-definite) matrix $C^{-1}$.

Beside the electrostatic energy it is assumed that electrons in each island occupy single particle quantum states (orbitals), whose energies $\epsilon_{i \alpha}(i=1,2, . . K ; \alpha=1,2, .$.$) depend on$ the confining potential as well as on the magnetic field. The latter is manifested through its orbital effects as well as due to Zeeman splitting (in which case the quantum number $\alpha$ contains also a spin label). The corresponding occupation numbers $n_{i \alpha}$ can be either 0 or 1 . The system described above might then be represented by a classical Hamiltonian

$$
H=H_{c}+H_{s p}
$$

where the charging Hamiltonian $H_{c}$ is just the electrostatic energy of (1) written in terms of the orbital occupation numbers

$$
H_{c}=\frac{1}{2} \sum_{i, j=1}^{K} p_{i j}\left[\sum_{\alpha} n_{i \alpha}\right]\left[\sum_{\alpha^{\prime}} n_{j \alpha^{\prime}}\right]-V_{g} \sum_{i=1}^{K} \sum_{\alpha} n_{i \alpha},
$$

and the single particle part of the Hamiltonian $H_{s p}$ is

$$
H_{s p}=\sum_{i=1}^{K} \sum_{\alpha} \epsilon_{i \alpha} n_{i \alpha} .
$$

The precise form of the matrix elements $p_{i j}=\left[C^{-1}\right]_{i j}$ as well as the single particle energies $\epsilon_{i \alpha}$ are specified in the next section when we present our results. Despite the fact that the Hamiltonian $H=H_{c}+H_{s p}$ is classical (and relatively simple), the elucidation of its spectrum for large $N$ and $K$ is virtually hopeless. In order to compute the ground state energy $E(N)$, one has to find the minimum of $H$ on all the possible $K$-tuples $\left(N_{1}, N_{2}, \ldots, N_{K}\right)$ with the 
constraint $\sum_{i=1}^{K} N_{i}=N$. Note that the so-called "Coulomb Glass" model obtains as a special case when $i$ refers to a lattice site with random energy $\epsilon_{i \alpha}=\epsilon_{i}$, and a single orbital $N_{i}=n_{i}=0,1$. The interaction matrix is then given by $p_{i j}=1 / r_{i j}$ for $i \neq j$ and $p_{i i}=0$, where $r_{i j}$ is the distance between sites $i$ and $j$.

The position of the $N^{t h}$ conductance peak is given by the first difference of the ground state energy, namely, the chemical potential of the (isolated) dot, $\mu \equiv E(N+1)-E(N)$. The spacing between peaks is determined by the second difference (the inverse compressibility),

$$
\chi_{N} \equiv E(N+1)-2 E(N)+E(N-1) .
$$

The occurrence of close peaks for certain values of electron number $N$ corresponds to small values of $\chi_{N}$ (recall that for a single island quantum dot, in which the single particle energies are neglected, the inverse compressibility is a constant $\left.1 / C_{11}\right)$. For the more general model described above the spacing distribution will of course fluctuate. In general, some constants appearing in the Hamiltonian $H=H_{c}+H_{s p}$ are random (e.g. the elements of the matrix $C$ and the single particle energies $\epsilon_{i \alpha}$ ), but most experiments are performed on a single quantum dot, so that fluctuations are meant with respect to the electron number $N$. The numbers $\chi(N)$ might then be considered as values assumed by a random variable $\chi$ which has a certain distribution function $P(\chi)$.

It might be instructive to compute the distribution $P(\chi)$ for a particular special case (albeit not realistic) which looks deceptively simple. The reader who is not interested may skip the rest of this section. Take $K>1, C_{i j}=0$ for $i \neq j$, and $\epsilon_{i \alpha}=0$. The Hamiltonian $H=H_{c}$ is the classical energy of a set of $K$ charged metallic bodies which are very far apart from each other, so there is no coupling among them. This problem then belongs to a class of problems dealing with statistics of spectra of independent quantum systems [10]. Here it refers to the addition spectra for a system composed of several independent subsystems, each of which has a certain spectrum which is a quadratic function of the number of particles residing on it. Thus, for example, by replacing $C_{i i}^{-1} \rightarrow \hbar \omega_{i}$, it can represent the energy of $N$ spinless fermions distributed among $K$ independent oscillators. 
Our aim is to find the distribution function $P(\chi)$. If charge is not quantized, the problem of finding the ground state energy is simply formulated as follows: Given $K$ independent capacitors $C_{i}, i=1, \ldots, K$, (in general, with random capacitances $C_{i}$ ), how should the corresponding charges $Q_{i}$ be chosen so as to minimize the electrostatic energy $E(Q)=$ $\sum_{i=1}^{K} \frac{Q_{i}^{2}}{2 C_{i}}$ while holding the total charge $Q=\sum_{i=1}^{K} Q_{i}$ fixed? This constrained minimum problem is trivially solved by requiring $\partial E(Q) / \partial Q=0$ and expressing $Q_{1}=Q-\sum_{i=2}^{K} Q_{i}$. It is evident from dimensional arguments that $E(Q)$ is proportional to $Q^{2}$, with some coefficient denoted by $1 / 2 C$, in which $C$ may be interpreted as the total capacitance of the system. Evidently, in this case the second derivative of $E(Q)$ with respect to $Q$ is independent of $\mathrm{Q}$ (and is easily calculated), namely $\partial^{2} E / \partial Q^{2}=1 / C$.

What happens then if charge is quantized? The formulation of the problem is now repeated albeit with $Q=N, Q_{i}=N_{i}$ (with $N$ and $N_{i}$ integers). Intuitively, one would expect $P(\chi)$ to be centered about $1 / C$, but, surprisingly, this is not the case, and $P(\chi)$ is large near $\chi=0$. An analytic expression for $P(\chi)$ has been obtained recently by the authors [11]. In order to present it, let us assume that the $K$ capacities $C_{i}, i=1,2, \ldots, K$, are rearranged so that $C_{1}>C_{2}>C_{3} \ldots>C_{K}$. It is also useful to divide all the capacitors by the largest one, to obtain scaled capacitors $c_{i} \equiv C_{i} / C_{1}$. with $1=c_{1}>c_{2}>c_{3}>\ldots>c_{K}$. Now let us define the following quantities:

$$
\begin{gathered}
a_{\ell}=\sum_{2 \leq i_{1}<i_{2}<. .<i_{\ell} \leq K} c_{i_{1}} c_{i_{2}} \ldots c_{i_{\ell}} \prod_{j \neq i_{m}}\left(1-c_{j}\right), \quad \ell=1,2, \ldots, K-1, \\
\mathcal{C} \equiv \sum_{i=1}^{K} c_{i} \\
B \equiv \prod_{i=2}^{K}\left(1-c_{i}\right) .
\end{gathered}
$$

With these notations we can provide a precise formula for the spacing distribution. The formula relates to the density function of the distribution and holds in the generic case (meaning that for a specific set of measure 0 of parameters it is incorrect). The following is 
obtained if the spacings are normalized to lie in the interval $[0,1]$ (multiplying all of them by $\left.C_{1}\right)$ :

$$
P(\chi)=\frac{1}{2 \mathcal{C}} \sum_{\ell=2}^{K} a_{\ell} \ell(\ell+1)(1-\chi)^{\ell-1}+\frac{B}{\mathcal{C}} \delta(1-\chi) .
$$

A plot of $P(\chi)$ is given in figure 1 and shows indeed that it has a maximum at $\chi=0$ and a delta function component at the inverse of the largest capacitance. In fact, when $K \rightarrow \infty$, the weight of the delta function shrinks to zero and (after an appropriate renormalization) $P(\chi)$ approaches the Poisson distribution $e^{-\chi}$.

\section{RESULTS}

We now return to the full Hamiltonian of Eq. 2. It contains the coefficients of capacitance and induction matrix $C_{i j}$ and the single particle energies $\epsilon_{i \alpha}$ as input. In choosing the actual numerical values we use a few guidelines, one of which is to avoid too many independent input data. As will be clear below, these are not fitting parameters, but rather a set of constants which are chosen once and for all on general physical grounds. First, for the electrostatic part, recall that the matrix $C$ should be a symmetric positive definite matrix with $C_{i i}>0$ and $C_{i j}<0$ for $i \neq j$. Besides, we expect it to be random. We then assume that $C_{i i}$ are random numbers uniformly distributed between 0 and $W$, whereas the nondiagonal elements are uniformly distributed between $-w$ and 0 . Actually, it is only the ratio $w / W$ which matters, so one may assume $W=1$ and use $e^{2} / W=1$ as an energy unit, leaving $w$ as a single constant reflecting the strength of coupling between islands. Practically, for each realization of $c_{i j}$ 's drawn at random we check whether the resulting matrix is positive definite, and reject realizations which are not. Thus, we must put the restriction $w<1$ in order to generate a positive definite matrix $C$. Indeed, for $w>0.25$ (and $K=5$ ) most of the matrices generated randomly failed to be positive definite. Second, for the single particle energies, we consider each electron island $i$ as a two-dimensional potential well 
$V_{i}(r)=\frac{1}{2} M \omega_{i}^{2} r^{2}$, where $M$ is an effective mass. One may then regard $\omega_{i}^{-1 / 2}$ as a measure of the radius of the corresponding electron island. Since the capacitance $C_{i i}$ is also proportional to this radius we assume $\omega_{i}=\gamma C_{i i}^{-2}$, where $\gamma$ is a constant reflecting the relation between charging energies and single particle energies. The single particle energies in each electron island (subject to a perpendicular magnetic field $B$ ) are then given explicitly. To be more specific, recall that in two dimensions there are two quantum numbers for the orbital motion (denoted hereafter by $n, m)$ to which we add a spin index $\sigma= \pm 1$. Then, with $\alpha=(n, m, \sigma)$, we have

$$
\epsilon_{i \alpha}=\frac{\hbar}{2}\left[n \omega_{c}+m \sqrt{\omega_{i}^{2}+\frac{1}{4} \omega_{c}^{2}}\right]+g \mu_{B} \sigma B,
$$

where $\omega_{c}=e B / M c$ is the cyclotron frequency, $g$ is the g-factor and $\mu_{B}$ is the Bohr magneton. Since $g$ contains the effective mass it is not known accurately. Its value is constrained on physical grounds (see below). Note that in this scheme the spacings between single particle energies are deterministic, and do not follow the Wigner surmise. The main cause of fluctuation is then due to the combination of non-random single particle energies and the occurrence of numerous charging energies. Finally, the number of electron islands $K$ is determined by the size of the quantum dot. The four input data of the model are then $K, w, \gamma$ and $g$. Note that the gate voltage $V_{g}$ does not have an important role here. Indeed, in actual experiments the variation of gate voltage serves to adjust the energies $E(N)$ with the chemical potential of the leads, but here the ground state energies are calculated directly. In order to avoid redundancy we are content with having a single set of these constants which is physically reasonable. In particular, it assures that the charging energy is much larger than single particle level spacings and that the Zeeman splitting is small at moderate magnetic fields. Specifically, for $B=0$ we test the distributions for $w=0.03,0.08$ and 0.20 , while for $B \neq 0$ we fix $w=0.03$. Besides, we take $\gamma=0.1$, and $g=0.0081$ is chosen such that for moderate fields the Zeeman splitting is of the order of the mean level spacing. Finally, the number of electron islands is fixed as $K=5$. Indeed, we use just a brute force trial algorithm, and hence cannot treat systems with a large number of electron islands. 
With these prescriptions the ground state energies $E(N)$ and their first and second differences are calculated for $B=0$ up to $N=200$ and for $B \neq 0$ up to $N=50$.

The first question we addressed is how the electrons are added among the islands. Figure 2 shows electron numbers $N_{i}$ in each of the five islands, v.s the total electron number $N$, in the range $60<N<80$ (for $w=0.03$ ). Evidently, the order of curves is according to the value of the capacitance $C_{i i}$. On a larger scale, the numbers $N_{i}$ grow linearly with $N$, as it should be. Let us then consider the question of redistribution. From figure 2 we see that redistribution occurs only once, as $N$ grows between 67 and 68 (see the vertical line). The addition of an electron to the second (or third) island involves also a transfer of an electron from the fourth island to the second (or the third) one. This scenario occurs also in other ranges of $N$ with the same proportion (namely about four percent). Thus, within the present model, redistribution is present, although it is rare and minimal.

The next question we address is how the ratio between the inductive and capacitive coefficients (represented here by the parameter $w$ ) affects the distribution $P(\chi)$. It was shown in the previous section that for $w=0$ the distribution has a maximum at $\chi=0$. It is intuitively expected (based on the concept of level repulsion) that for $w \neq 0$ the maximum will be shifted away from zero. This is also verified by our numerical results but, somewhat unexpectedly, the peak at small $\chi$ persists even at $w=0.20$. The distribution $P(\chi)$ is drawn in figure 3 for $w=0.03,0.08$ and $w / W=0.20$. The normalization is such that the largest capacitor is $C_{11}=1.0$, so that if other capacitors are absent the distribution would have a delta function at $\chi=1$. In all three cases the peak position is much smaller than 1 . Recall, however, that besides the capacitance induction matrix $C$ the total energy is determined also by the single particle energies in each island. The remarkable point is that the distribution is not Gaussian (which is the hallmark of spacing distributions in small quantum dots). Finally, we check the behavior of the first difference $E(N+1)-E(N)$ as a function of the magnetic field. Recall that this quantity is proportional to the position of the $N^{\text {th }}$ Coulomb blockade peak. As a measure of the strength of the magnetic field we use the parameter $\omega_{c} / \omega_{0}$, where $\omega_{0}$ is the harmonic oscillator frequency of the largest island. The positions 
of the peaks for $39<N<48$ and $w / W=0.03$ are displayed in figure 4 . A comparison with the results displayed in figure 2 of Ref. [7] indicates a remarkable qualitative agreement. In particular, it shows that groups of two (and sometimes even three) electrons can tunnel through the quantum dot at almost the same gate voltage. The oscillations at small magnetic field just mark transitions to lower Landau levels as the magnetic field increases. The phenomena of alternate bunching $(N, N+1) \rightarrow(N-1, N)$ is also reproduced in the present picture.

In conclusion, we suggest a classical model in which a large semiconductor quantum dot is viewed as a collection of metallic electron islands with capacitive and inductive coupling among them. The effect of adding a magnetic field is manifested through its orbital as well as its spin contents. The model can explain the occasional occurrence of couples or even triples of closely spaced Coulomb blockade peaks, as well as the qualitative behavior of peak positions with the magnetic field. The results of the previous section displayed in figure 1 together with an analysis of the results displayed in figure 3 can provide an answer to the question why, in the present model, there are couples and sometimes even triples of close Coulomb blockade peaks. When the capacitors are completely independent, the distribution is close to Poissonian as can be proved analytically. Introducing an inductive coupling spoils this picture, but does not destroy it completely, so that even at $w=0.2$ there is still a probability for the occurrence of close peaks. The eventual decrease of the distribution near $\chi=0$ seems to be related to the fact that, unlike the case of independent systems, the capacitance matrix is non-diagonal. Switching on the coupling here then has an effect similar to a weak "spacing repulsion", similar to the familiar effect of perturbation in two level systems.

\section{ACKNOWLEDGMENTS}

This research was supported in part by a grant from the Israel Academy of Science and Humanities under Centers of Excellence Program. One of us (Y. A) is grateful to R. Ashoori 
for discussion and suggestions. 
[1] U. Sivan, R. Berkovits, Y. Aloni, O. Prus, A. Auerbach and G. Ben Yoseph, Phys. Rev. Lett. 77, 1123 (1996); F. Simmel, T. Heinzel, and D. A. Wharam, Europhys. Lett. 38, 123 (1997); S. R. Patel, S. M. Cronenwett, P. R. Stewart, A. G. Huibers, C. M. Marcus, C. I. Duruo, J. S. Harris, K. Campman and A. C. Gossard, Phys. Rev. Lett. 80, 4522 (1998).

[2] L. P. Kouwenhoven, T. H. Oosterkamp, M. W. S. Danoesastro, M. Eto, D. G. Austing, T. Honda and S. Tarucha, Science 278, 1788 (1997).

[3] R. Berkovits, Phys. Rev. Lett. 81, 2128 (1998); R. Berkovits and B. L. Altshuler, Phys. Rev. B 55, 5297 (1997); Ya. M. Blanter, A. D. Mirlin and B. A. Muzykantskii, Phys. Rev. Lett. 78, 2449 (1997); A. A. Koulakov, F. G. Pikus and B. I. Shklovskii, Phys. Rev. 54, 9223 (1997).

[4] D. Orgad and S. Levit, unpublished.

[5] R. C. Ashoori et al., Phys. Rev. Lett. 68, 3088 (1992).

[6] R. C. Ashoori et al., Physica (Amsterdam) 189B, 117 (1993).

[7] N.B. Zhitenev, R.C. Ashoori, L.N. Pfeiffer and K.W. West, Phys. Rev. Lett. 79(1997), 2308.

[8] Y. Wan, G. Ortiz and P. Phillips, Phys. Rev. Lett. 75, 2879 (1995); Phys. Rev. B 55, 5313 (1997); Phys. Rev. Lett. 78(C), 3979 (1997).

[9] M. E. Raikh, L. I. Glazman and L. E. Zhukov, Phys. Rev. Lett. 77, 1354 (1996); Phys. Rev. Lett. 78(C), 3980 (1997).

[10] M. V. Berry and M. Tabor, Proc. Roy. Soc. London 356, 375 (1977)

[11] Y. Avishai, D. Berend and R. Berkovits, Journal of Physics A (Math. Gen.) 31, 8063 (1998). 


\section{FIGURE CAPTIONS}

FIG. 1. Distribution of inverse compressibility $P(\chi)$ for a system containing independent subsystems each of which has a ground state energy proportional to the square of the number of particles it contains. The graph corresponds to a special case of equation 9 with $K=5$.

FIG. 2. Distribution of electrons among the five islands as a function of the total electron number $N$ at zero magnetic field. The choice of constants $K=5, w=0.03$ is explained in the text. The number $N_{i}$ of electrons on island $i$ is commensurate with its capacitance $C_{i i}$. In most cases the addition of an electron does not perturb the occupation of other islands. An example of redistribution is marked with a vertical line. An addition of an electron causes a minimal redistribution.

FIG. 3. Effect of strength of inductive coupling $w$ on the distribution $P(\chi)$ of level spacings in the dot at zero magnetic field (not normalized). It is displayed for $K=5$ and $w=0.03,0.08$ and 0.20 in (a), (b) and (c) respectively.

FIG. 4. Coulomb blockade peak position for electron number $N$ between 39 and 48 as functions of the magnetic field. Here $\omega_{o}$ is the oscillator frequency of the largest island and $\omega_{c}$ is the cyclotron frequency. The units on the ordinate are energy units as explained previously. They are proportional to the gate voltage appropriate for the corresponding peak. The values chosen for the constants $w=0.03, K=5, \gamma=0.1$ and $g=0.0081$ are explained in the text. 


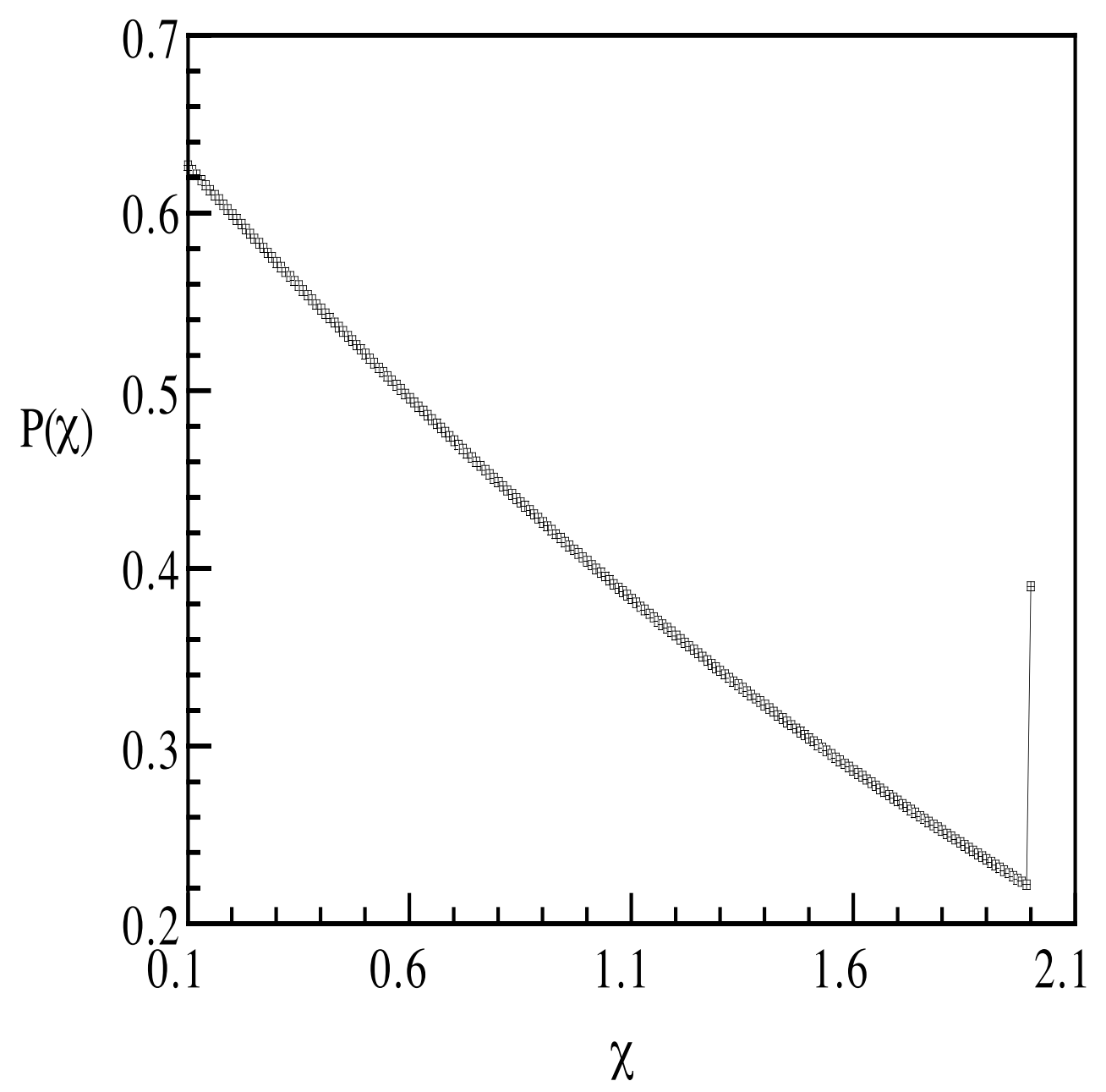




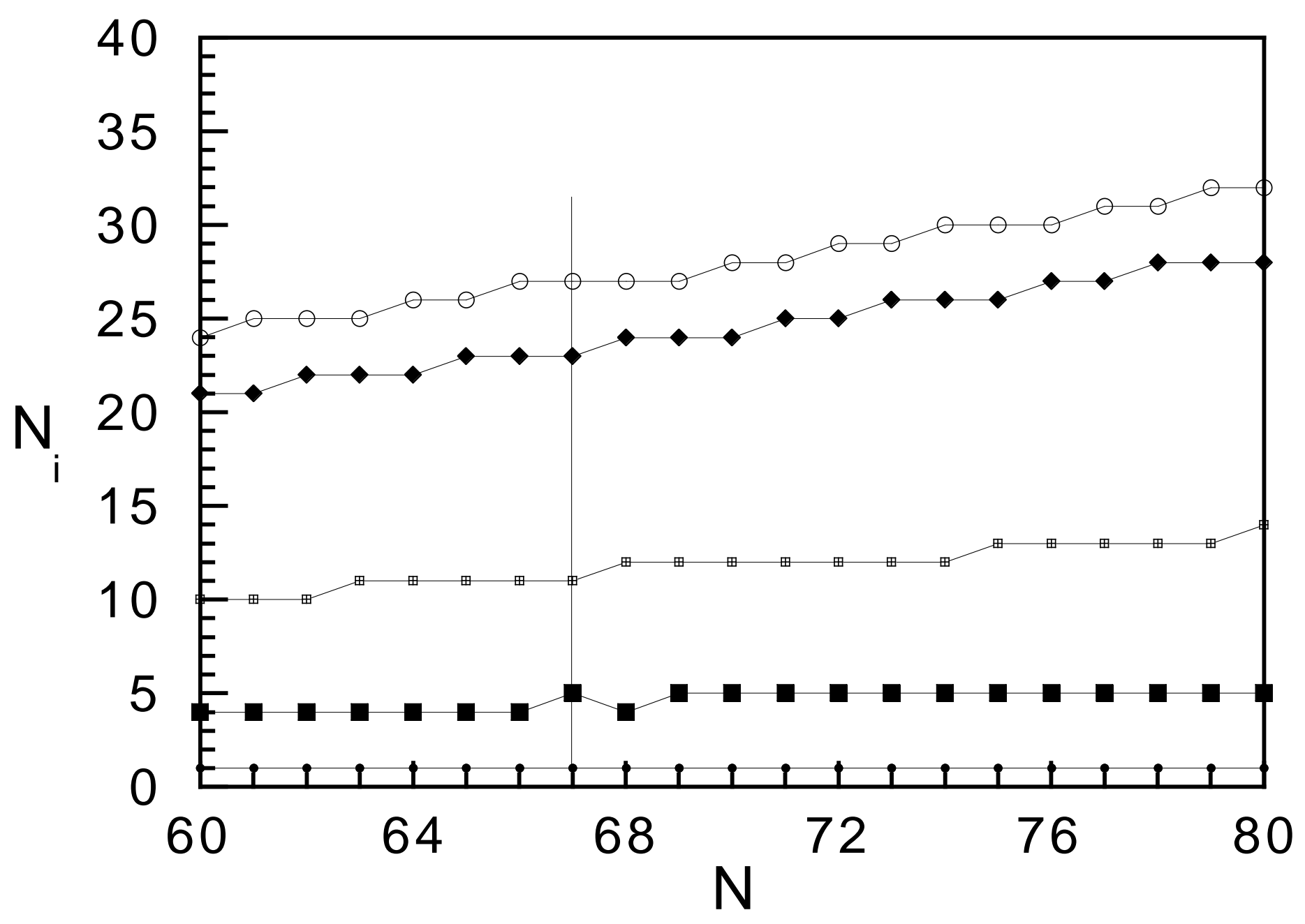



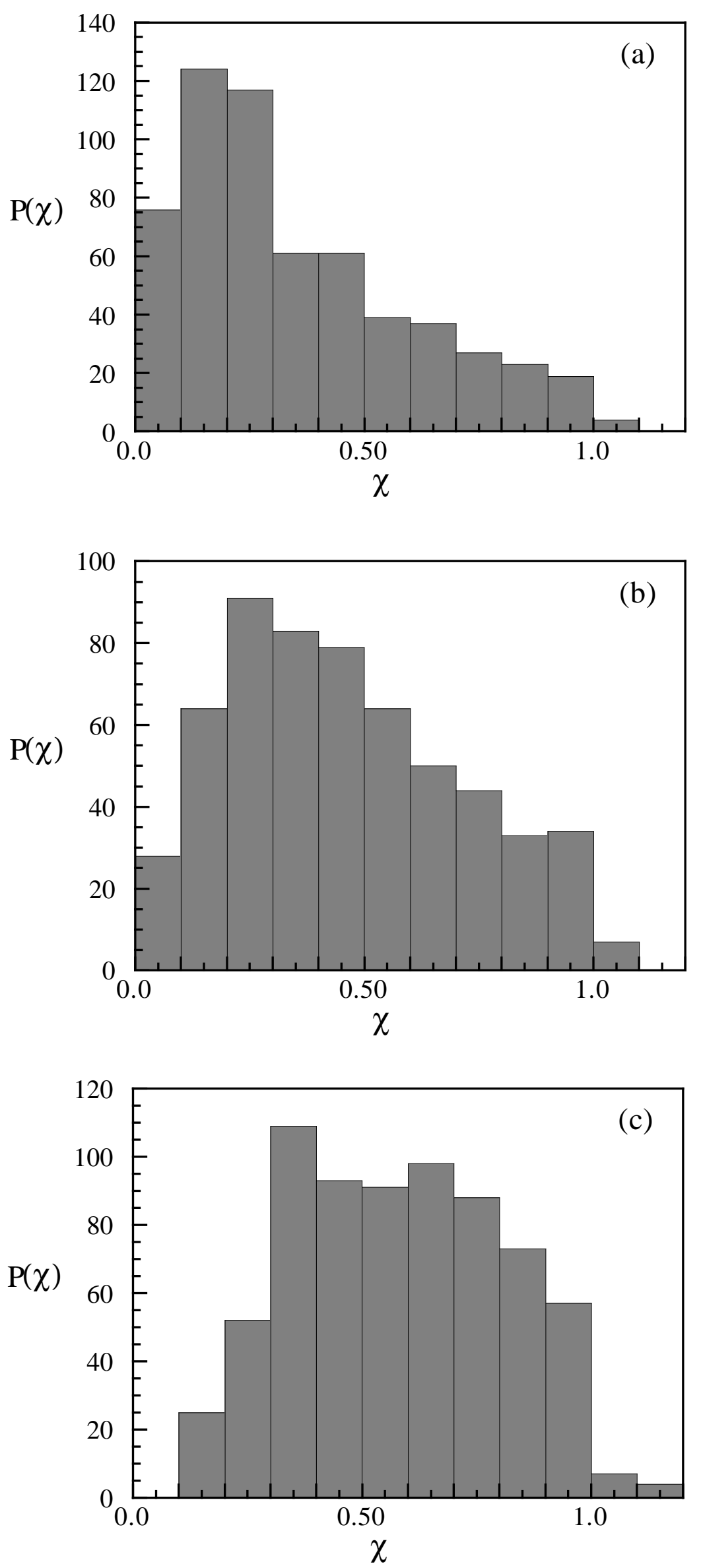


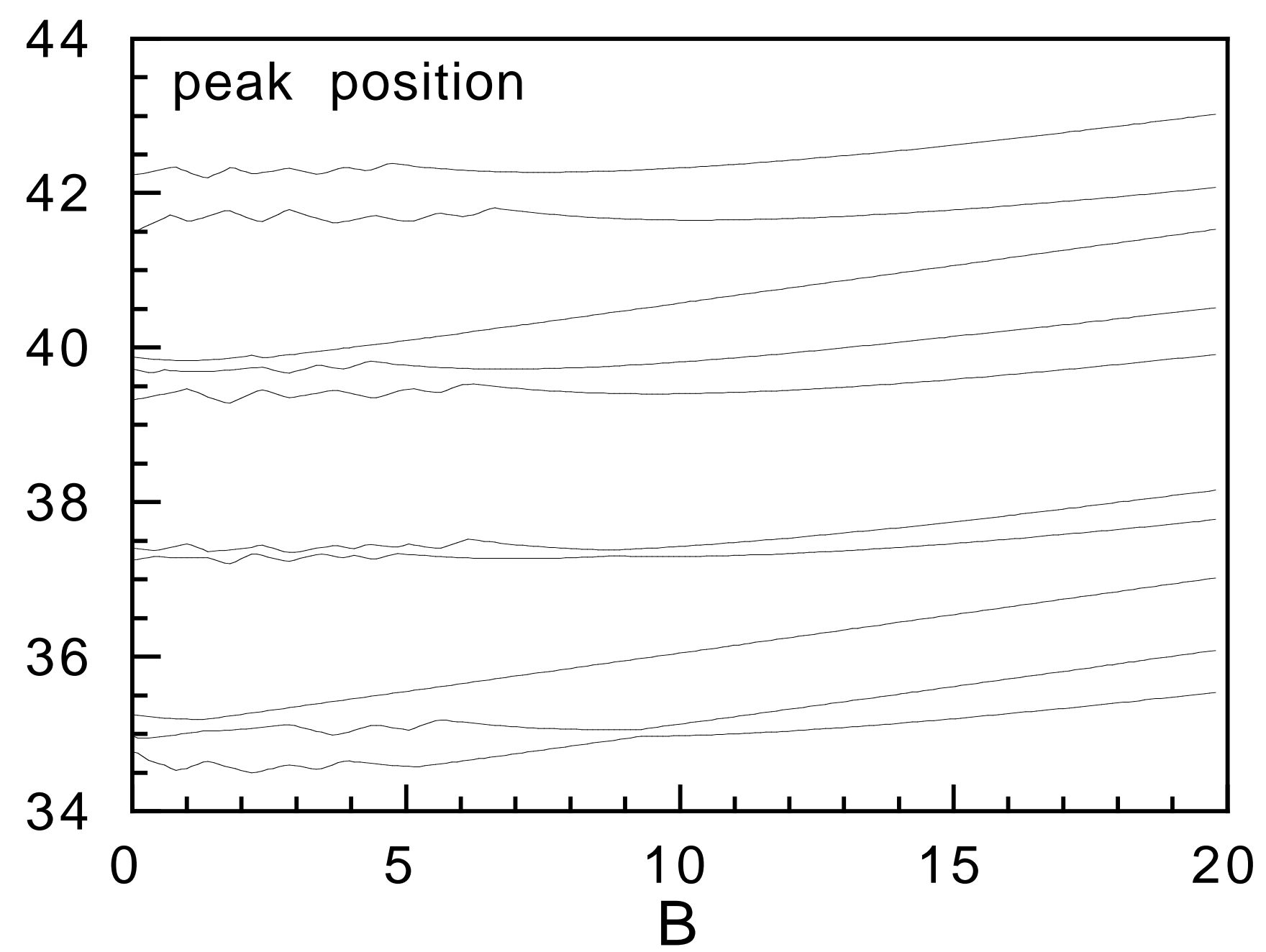

